\title{
Instrumentation pour I'optique du rayonnement X
}

\author{
Jean-Michel ANDRÉ et Philippe JONNARD \\ Laboratoire Chimie Physique - Matière Rayonnement, UPMC Université Paris 06, CNRS UMR 7614, \\ 11 rue Pierre et Marie Curie, 75231 Paris Cedex 05 \\ jean-michel.andre1@upmc.fr
}

$L^{\prime}$ optique du rayonnement $X$ est conditionnée par le fait que l'indice optique est un nombre complexe dont la partie réelle (indice de réfraction) est légèrement inférieure à l'unité et dont la partie imaginaire n'est pas négligeable. Autrement dit, les matériaux sont peu réfringents et peu transparents à ce rayonnement. Du fait que l'indice de réfraction est légèrement inférieur à 1 , la réflexion spéculaire par un miroir homogène ne peut se faire efficacement que dans les conditions de réflexion totale externe (RTE) pour un angle d'incidence supérieur à l'angle critique, c'est-à-dire en pratique sous incidence rasante (voir encadré). La faible réfringence et l'opacité des matériaux empêchent la réalisation de lentilles semblables à celles mises en œuvre pour le rayonnement visible. De ces contraintes, il découle que les dispositifs optiques destinés à l'imagerie et à la dispersion spectrale du rayonnement $X$ sont des instruments souvent très élaborés qui font appel à des technologies de pointe.

$$
\mathrm{L}
$$
'optique du rayonnement $\mathrm{X}$ est conditionnée par le fait que l'indice optique est un nombre complexe dont la partie réelle (indice de réfraction) est légèrement inférieure à l'unité et dont la partie imaginaire n'est pas négligeable. Autrement dit, les matériaux sont peu réfringents et peu transparents à ce rayonnement.

Du fait que l'indice de réfraction est légèrement inférieur à 1 , la réflexion spéculaire par un miroir homogène ne peut se faire efficacement que dans les conditions de réflexion totale externe (RTE) pour un angle d'incidence supérieur à l'angle critique, c'est-à-dire en pratique sous incidence rasante (voir encadré). La faible réfringence et l'opacité des matériaux empêchent la réalisation de lentilles semblables à celles mises en œuvre pour le rayonnement visible. De ces contraintes, il découle que les dispositifs optiques destinés à l'imagerie et à la dispersion spectrale du rayonnement $X$ sont des instruments souvent très élaborés qui font appel à des technologies de pointe.

\section{Le rayonnement $X$ et ses systèmes optiques}

Le rayonnement $X$ couvre un large domaine du spectre électromagnétique ; dans cet article nous considérerons qu'il s'étend de plusieurs centaines de keV à $50 \mathrm{eV}$. La partie du spectre correspondant aux photons $X$ de plus de $5 \mathrm{keV}$ constitue les rayons $X$ durs tandis que la partie cor- respondant aux photons d'énergie inférieure à $5 \mathrm{keV}$ constitue le rayonnement $X$ mou. Nous parlerons aussi de rayonnement extrême UV (EUV) pour le domaine d'énergie inférieure à $100 \mathrm{eV}$.

Le diagramme donné dans la figure 1 donne un tableau (non exhaustif) des différents systèmes optiques utilisés en optique $X$. Nous passerons en revue les différents types de systèmes optiques suivant le phénomène qu'ils mettent en œuvre: réflexion totale, diffraction, réfraction et absorption. Soulignons qu'il existe des systèmes « hybrides » qui utilisent plusieurs phénomènes comme les lentilles de Bragg-Fresnel ou les réseaux de diffraction multicouches. Peuvent être aussi qualifiés d'hybrides des systèmes multimiroirs fonctionnant dans les conditions

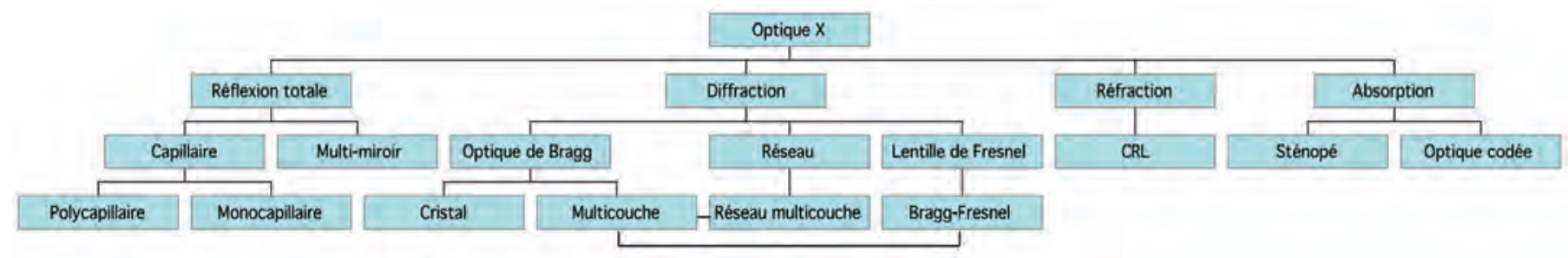

Figure 1. Diagramme des différents systèmes optiques utilisés dans le domaine des rayons $X$. 
de Bragg avec des revêtements multicouches interférentiels.

\section{Systèmes à réflexion totale externe (RTE)}

\section{Multi-miroirs}

Les systèmes multi-miroirs sont destinés à l'imagerie et ont souvent été mis en œuvre dans les télescopes pour l'astrophysique et dans les microscopes X. Ils comprennent des miroirs courbés elliptiquement, hyperboliquement ou paraboliquement suivant différents arrangements; on peut citer les optiques de Kirpatrick-Baez (KB, 1948), de Wolter (1952) et de Montel (1957). Bien que ces systèmes soient de conception relativement ancienne (la date de leur invention est indiquée entre parenthèses), ils sont largement utilisés dans les instruments récents et certains ( $\mathrm{KB}$ et Montel) sont même déclinés en version réflexion de Bragg avec des revêtements multicouches.

En version de base RTE, les substrats sont généralement revêtus de matériaux lourds ( $\mathrm{Au}, \mathrm{Pt}, \mathrm{Rh}, \mathrm{Pd}$ ) offrant un pouvoir réflecteur important et un angle d'attaque critique élevé. Ces instruments sont délicats à réaliser car ils nécessitent des performances en termes de forme et de qualité de surface (rugosité) difficiles à atteindre. Les montages Wolter sont à la base des télescopes spatiaux Chandra et XMM-Newton. Les télescopes à rayons $X$ ont permis de découvrir de nombreux objets célestes émettant du rayonnement $X$ (restes de supernova, systèmes binaires...). Rappelons que Riccardo Giacconi est considéré comme le père de ces télescopes et a obtenu le prix Nobel de physique en 2002 pour la découverte de nombreuses sources cosmiques de rayons $X$.

\section{Optique « Lobster »}

Il s'agit d'une variante du système à multi-miroirs inspiré de l'œil des crustacés décapodes, en particulier le homard (d'où le nom d'optique «Lobster»). Cette opti que consiste en une grille de microcanaux de section carrée dont l'entrée est située sur une surface sphérique, les canaux visant le centre de la sphère. Le rayonnement émis par une source ponctuelle est focalisé sur une tâche focale assez étendue par rapport à la source. Ces optiques offrent un champ très large; leur principale application est l'exploration du ciel pour détecter des sources $X$ célestes.

\section{Capillaires}

Les optiques à capillaires utilisent la RTE sur la surface interne de la paroi de capillaires en verre ; il existe des systèmes mono- et poly-capillaires. Les systèmes poly-capillaires, dits de Kumakhov, sont formés d'un faisceau de mono-capillaires. Il ne s'agit pas d'optique d'imagerie à proprement parler mais plutôt d'optique d'illumination permettant d'éclairer un point focal à partir d'un point source divergent ou d'optique de collimation permettant d'obtenir un large faisceau quasi parallèle à partir d'une source divergente. Des gains en intensité de plusieurs milliers peuvent être obtenus et les diamètres de tâche focale sont compris entre quelques dixièmes et quelques milliers de micromètres. Les optiques de type focalisant sont très largement utilisées dans les systèmes de microanalyse nécessitant des micro-faisceaux intenses (micro-fluorescence, micro-diffractométrie...) tandis que les optiques de collimation ont par exemple été utilisées pour la lithographie $X$, les mesures de contrainte des « wafers» en microélectronique et la cristallographie de protéines.

\section{Systèmes diffractifs}

\section{Optique de Bragg}

Ces optiques utilisent les conditions d'interférences constructives pour réfléchir, focaliser ou disperser le rayonnement $X$. Contrairement aux systèmes à RTE qui sont pratiquement achromatiques, ces systèmes diffractifs sont très chromatiques. On distingue les systèmes périodiques qui fonctionnent selon la diffraction de Bragg et les systèmes à zone de Fresnel.

En diffraction de Bragg, les cristaux naturels ont été employés dès le début de la physique du rayonnement $X$ avec les travaux de Laue et Bragg, pour disperser spatialement la partie dure du spectre ; pour la partie molle du spectre ont été développées les couches de LangmuirBlogett, puis dans les années 70 les structures multicouches. Bien que des dispositifs d'imagerie par diffraction cristalline aient été réalisés avec succès en particulier

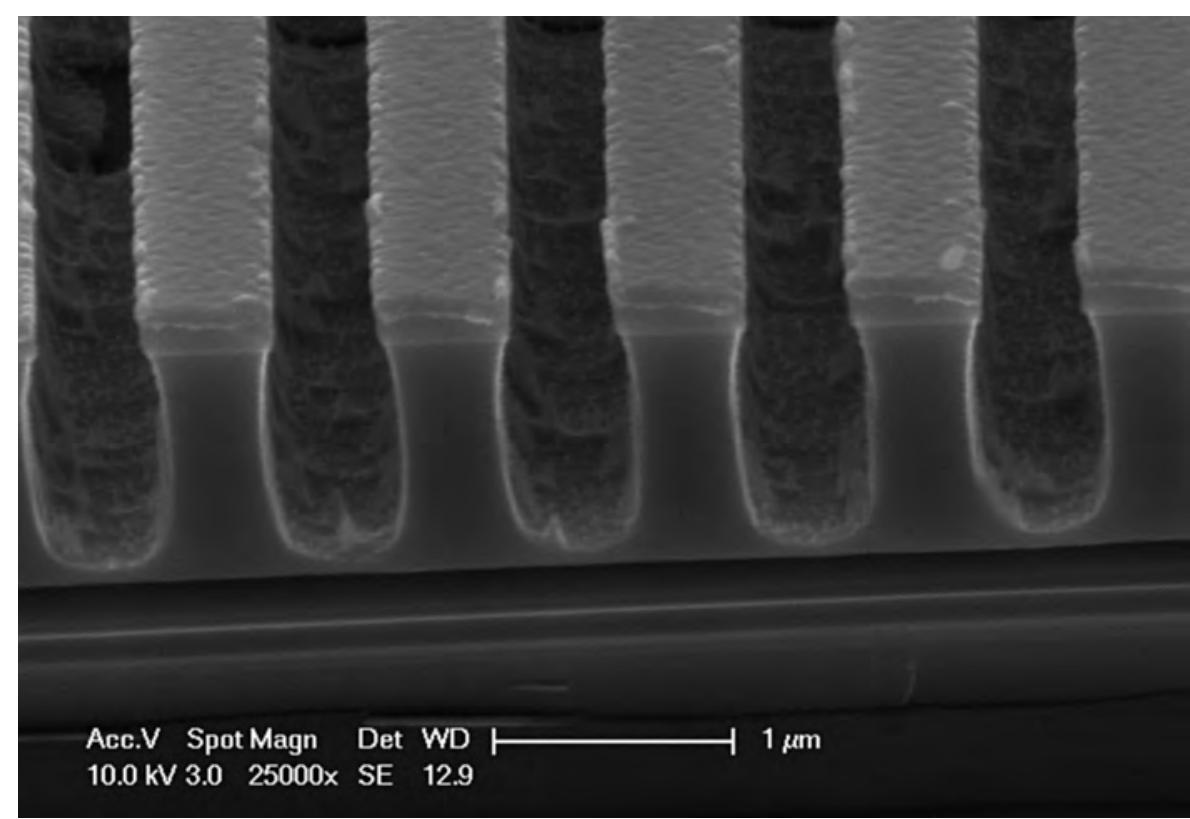

Figure 2. Image en microscopie électronique à balayage d'une multicouche gravée selon le profil d'un réseau lamellaire, vue de profil. La période du réseav est de $1 \mu \mathrm{m}$. L'épaisseur des barres de multicouches Mo/B4C est en moyenne de $400 \mathrm{~nm}$. 
par Montel dans les années 60, celle-ci a été essentiellement utilisée pour la spectroscopie $X$ qui a connu un développement considérable avec les travaux de $Y$. Cauchois en France. Par contre, compte tenu de leur bande passante relativement large et de la possibilité de les déposer sur des surfaces courbes, les structures multicouches ont été largement employées à des fins d'imagerie : il s'agit des systèmes hybrides multicouches. Ainsi l'imageur extrême ultraviolet EUVI de la mission STEREO lancée en 2006 comprend un télescope de type Ritchey-Chrétien à quatre voies (quadrants) comportant un miroir multicouche Mo/Si spécifique permettant de diffracter plusieurs émissions solaires du fer, Fe IX, FeX, Fe XII... et l'émission He II de l'hélium.

Les miroirs multicouches sont aussi largement mis en œuvre dans les spectromètres à rayons $X$ mous. Ils permettent l'analyse des éléments chimiques légers (du béryllium au fluor). Malheureusement la largeur de leur bande passante affecte considérablement leur résolution spectrale. Des structures multicouches gravées selon le profil des réseaux lamellaires ont été développées et employées avec succès pour réduire la bande passante (figure 2).

La possibilité de réfléchir le rayonnement $X$ dans les conditions de Bragg au voisinage de l'angle de Brewster permet d'utiliser les structures multicouches dans des instruments où intervient la polarisation du rayonnement : des polarimètres à rayons $X$ mous ont été implantés sur plusieurs synchrotrons tels que BESSY II ou ELETTRA. Il est aussi possible de réaliser des sources de rayonnement polarisé ; ainsi la NASA a-t-elle lancé en 2010 un projet pour de telles sources dans le cadre de l'étude de la polarisation du rayonnement $X$ émis par des sources spatiales. Cette recherche est citée comme une des priorités pour l'astronomie du XXI ${ }^{\mathrm{e}}$ siècle dans un rapport du National Research Council américain.
Le dépôt multicouche peut aussi être réalisé sur des membranes fines, quelques centaines de nanomètres de nitrure de silicium par exemple, pour réaliser des structures semi-transparentes ou même être autosupporté. Ces structures peuvent être employées comme lame séparatrice en interférométrie, analyseur pour spectrométrie par transmission, retardateur de phase, etc.

Sur le plan économique, le plus grand impact de ces structures va probablement concerner la lithographie EUV pour la microélectronique : la prochaine génération de masqueurs devrait employer non plus du rayonnement UV profond à la longueur d'onde $193 \mathrm{~nm}$ mais du rayonnement EUV à la longueur d'onde 13,5 nm où tous les éléments optiques, y compris le photo-masque, seront constitués de miroirs $\mathrm{Mo} / \mathrm{Si}$ dont la réflectivité pic à incidence normale avoisine $70 \%$. Les systèmes de pré-production actuels contiennent au moins deux miroirs condenseurs, six miroirs de projections et le masque

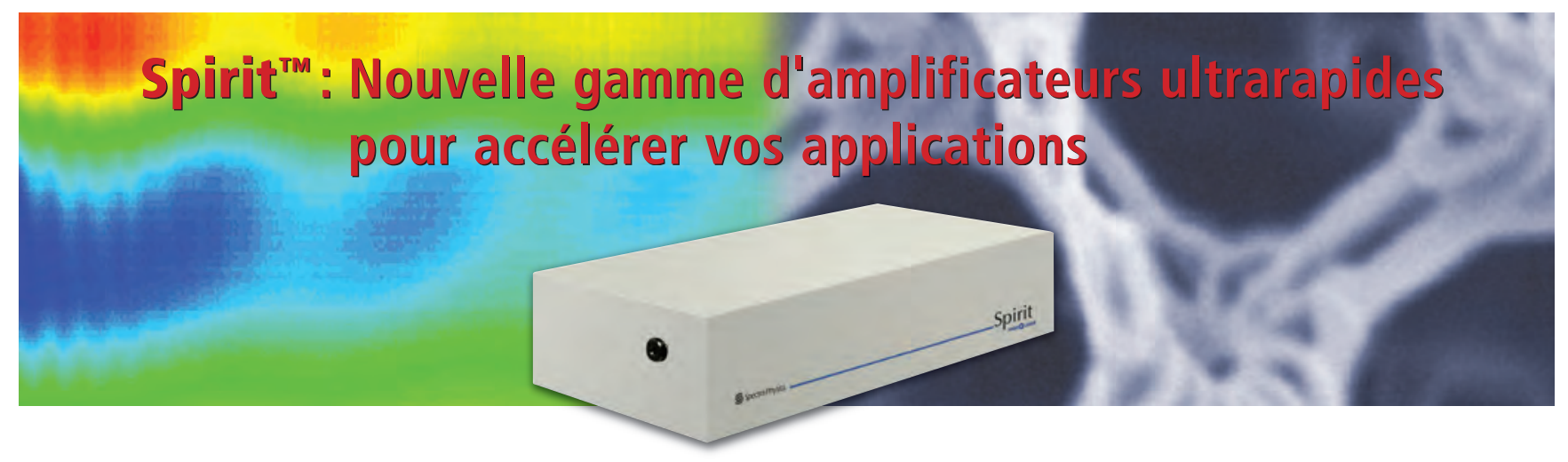

Spectra-Physics présente sa nouvelle gamme d'amplificateurs ultrarapides tout-en-un Spirit ${ }^{\text {TM }}$ à haut taux de répétitivité et réglable. Basée sur la technologie de pompage direct par diodes, développée par High Q Lasers (désormais intégrée à Spectra-Physics), I'architecture simple et novatrice de la gamme Spirit délivre des impulsions femtosecondes nécessaires au micro-usinage de composants médicaux et autres matériaux, ainsi qu'à la nanostructuration et aux applications scientifiques à résolution temporelle sélectionnée. Avec les amplificateurs Spirit, accélérez vos applications et obtenez des résultats rapidement.

Acteur majeur de l'innovation, Spectra-Physics se positionne une fois de plus comme le leader du marché des solutions ultrarapides en proposant la gamme de technologies la plus vaste, les champs d'application les plus variés et la base installée la plus étendue du secteur.

Pour plus d'informations sur la gamme Spirit, consultez www.newport.com/spirit ou appelez-nous.

MICRO-CONTROLE Spectra-Physics S.A.S

$\begin{array}{ll}\text { 1, rue Jules Guesde - Bâtiment B } & \text { Tél.: 01.60.91.68.68 } \\ \text { Zl. du Bois de l'Épine - BP189 } & \text { Fax: 01.60.91.68.69 }\end{array}$

91006 Évry CEDEX e-mail : france@newport.com
Spectra-Physics. \#1 in Ultrafast.

Expertise - Innovation - Results
Spectra-Physics.

une marque de Newport Corporation

으 2012 Newport Corporation. 
multicouche. En 2011 la société ASML a livré la première série de machines à lithographie EUV.

Au-delà de la «pure» diffraction de Bragg, des miroirs multicouches apériodiques ont été développés. Ils permettent soit de travailler sous plus faible incidence (en diminuant la valeur de l'angle d'incidence critique par rapport à un miroir homogène), pour des applications en astrophysique ou sur les lignes synchrotrons pour le rayonnement $X$ très dur, soit de réfléchir des impulsions ultra-brèves dites "attoseconde» de rayonnement EUV (miroirs chirpés).

\section{Réseaux de diffraction}

Les réseaux de diffraction sont utilisés avec le rayonnement mou et principalement comme élément dispersif des monochromateurs dans les lignes de lumière pour le rayonnement synchrotron. Ils fonctionnent généralement en mode RTE sous grande incidence avec des efficacités peu élevées. En standard les principaux manufacturiers proposent des réseaux à profil laminaire à utiliser en incidence oblique; ils sont produits en combinant une exposition holographique et la technologie de gravure par faisceau d'ions. Les monochromateurs utilisent des réseaux plans comme dans le système FLIPPER, sphériques comme dans le fameux montage "Grasshopper» ou toroïdaux. Les récents progrès concernent les réseaux à pas variable RPV et les réseaux hybrides à multicouche RHM. Les RPV permettent de focaliser le rayonnement dispersé sans courbure du substrat (mode Harada de focalisation) mais ils offrent surtout l'avantage de construire des instruments exempts d'aberration; ils peuvent être réalisés par des techniques holographiques. Des résolutions considérables avec des efficacités élevées peuvent être obtenues avec les RHM les plus modernes; ainsi la réalisation d'un réseau blasé de 10000 traits par $\mathrm{mm}$ recouvert d'une multicouche $\mathrm{Al} / \mathrm{Zr}$ offrant une efficacité de $24 \%$ au premier ordre à la longueur d'onde 17,2 nm vient d'être publiée en 2011 par une équipe russo-américaine.

\section{Lentilles de Fresnel}

Les lentilles de Fresnel sont basées sur le principe des interférences constructives entre ondes diffusées par les zones isophases (elliptiques) du système diffractant. Depuis les travaux de pionnier de Baez, les lentilles par transmission ont été largement utilisées comme composants (condenseur et objectif) dans les microscopes $X$ travaillant en mode imagerie ou balayage. Afin de s'affranchir du fond parasite au foyer primaire, des lentilles à modulation de phase ont été dévelop-

\section{La réflexion totale}

L'indice de réfraction (partie réelle de l'indice optique) des matériaux est généralement inférieur à l'unité dans le domaine des rayons $X$. L'indice du vide est égal à 1 . II s'ensuit que si l'angle d'incidence du rayonnement incident est plus grand qu'un certain angle cri- tique, alors il n'y aura pas réfraction à l'intérieur du matériau. Par conséquent le rayonnement sera totalement réfléchi. Dans la figure ci-dessous, i est l'angle d'incidence et de réflexion, $i_{r}$ l'angle de réfraction et $i_{c} I^{\prime}$ angle de critique de réflexion totale.
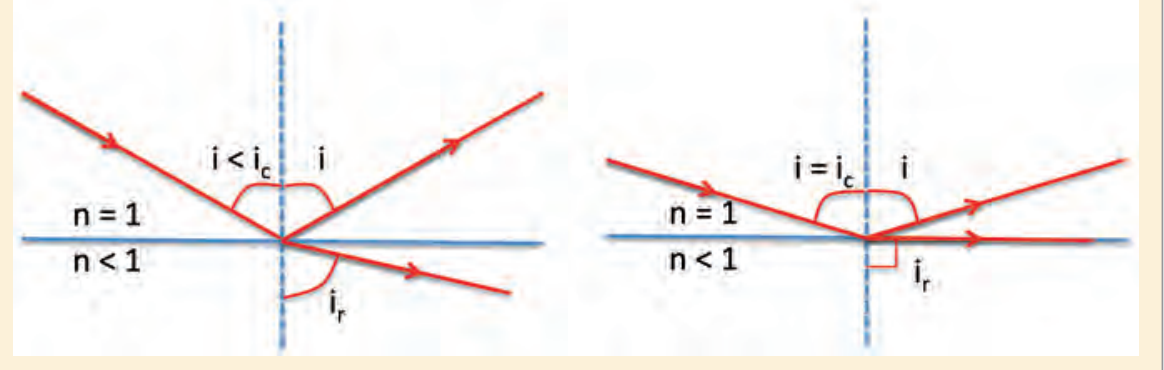

pées à partir des travaux de Kirz: au lieu de transmettre ou d'absorber le rayonnement, les zones changent la phase d'une quantité déterminée. Ces lentilles sont actuellement très élaborées et utilisables dans I'EUV : en 2011 une lentille de phase avec des zones de molybdène déposées sur une membrane de nitrure de silicium et ayant un diamètre de la zone la plus externe de $4 \mathrm{~mm}$ a été réalisée pour le domaine de longueur d'onde compris entre 7 et $18,5 \mathrm{~nm}$ pour permettre la métrologie de la luminosité du Soleil.

Des systèmes hybrides appelés lentilles de Bragg-Fresnel combinant la diffraction de Bragg par des cristaux ou des multicouches et le principe des zones de Fresnel, ont commencé à être développés dans les années quatre-vingt. Les lentilles de Bragg-Fresnel ont été employées d'une manière extensive auprès des synchrotrons de $3^{\mathrm{e}}$ génération pour la microscopie et la micro-diffraction $X$, en particulier dans le domaine des $X$ durs et comme imageur dans le système de diagnostic des plasmas.

\section{Systèmes à réfraction}

La réfraction au niveau d'un dioptre étant très faible dans le domaine des rayons $X$, il est nécessaire pour réaliser une lentille réfractive offrant une déviation suffisante des rayons, d'associer un nombre important d'éléments réfractifs. Ce fait a conduit à la réalisation de lentilles réfractives composées (CRL en anglais). Plusieurs systèmes ont été testés:

- Développées à partir de 1996, les lentilles à réseau de trous : des trous réalisés dans une barre de béryllium ou d'aluminium agissent comme des lentilles cylindriques; le principal inconvénient de ces lentilles est leur forte aberration sphérique.

- Développées à partir de 1998, les lentilles à réseau de bulles de gaz : des centaines de bulles de gaz sont alignées dans un capillaire rempli de résine époxy ; elles présentent le même inconvénient que les lentilles à trous.

- Développé à partir de 2000, le système "Alligator» constitué par une série de micro-prismes alignés sur un plan en 
regard d'une série identique pour former une structure en forme de mâchoire d'alligator. Les sillons de deux disques vinyl font l'affaire : système plutôt performant au regard du coût !

Plus récemment, d'autres montages dans le même esprit ont été réalisés, comme les lentilles à prisme enroulées.

\section{Systèmes à absorption}

\section{Caméra à sténopé}

Son principe est à la base des premiers instruments optiques d'imagerie : camera obscura attribuée à Della Porta au XVIe siècle. Souffrant d'une faible luminosité et de divers problèmes tels que la diffraction par le trou et l'effet de vignettage, son emploi est très limité en pratique.

\section{Optique à ouverture codée}

Ce type de système d'imagerie consiste à transmettre partiellement le rayonnement, au moyen d'un masque opaque dans lequel on a pratiqué des ouvertures suivant un schéma connu. L'ombre codée formée sur le détecteur est ensuite analysée par un algorithme pour tenter de reconstituer la source. Différents types de masque (URA, MURA, Levin...) ont été développés et offrent une résolution, une sensibilité, une réjection du bruit de fond, une simplicité de traitement algorithmique, etc. différentes. Ces optiques sont surtout employées pour le rayonnement $X$ très dur voire gamma qui est très difficile à dévier, et ont trouvé leurs principales applications en astronomie et en tomographie. Le satellite européen INTEGRAL lancé en 2002 et toujours en fonctionnement, dispose de plusieurs optiques codées pour analyser (par imagerie et spectrométrie) les sources célestes jusqu'au rayonnement gamma.

\section{Des instruments originaux}

Pour s'affranchir des contraintes imposées par la propagation du rayonnement $X$ dans la matière, les opticiens doivent faire preuve d'une grande imagination et développer des instruments originaux souvent très différents de ceux employés en optique visible. Les composants optiques implantés dans ces instruments requièrent de plus en plus la maîtrise de technologies de pointe.

\section{Pour en savoir plus}

"Soft x-rays and extreme ultraviolet radiation: Principles and Application», D. Attwood, Cambridge University Press (1999).

«X-ray multilayer optics : A review», J.-M. André et R. Barchewitz, Recent Res. Devel. Optics, vol. 2 (2002). "Modern Developments in X-ray and Neutron Optics», Springer Series in Optical Science N N 137, A. Erko, M. Idir, T. Krist, A.G. Michette, Eds. (2008).

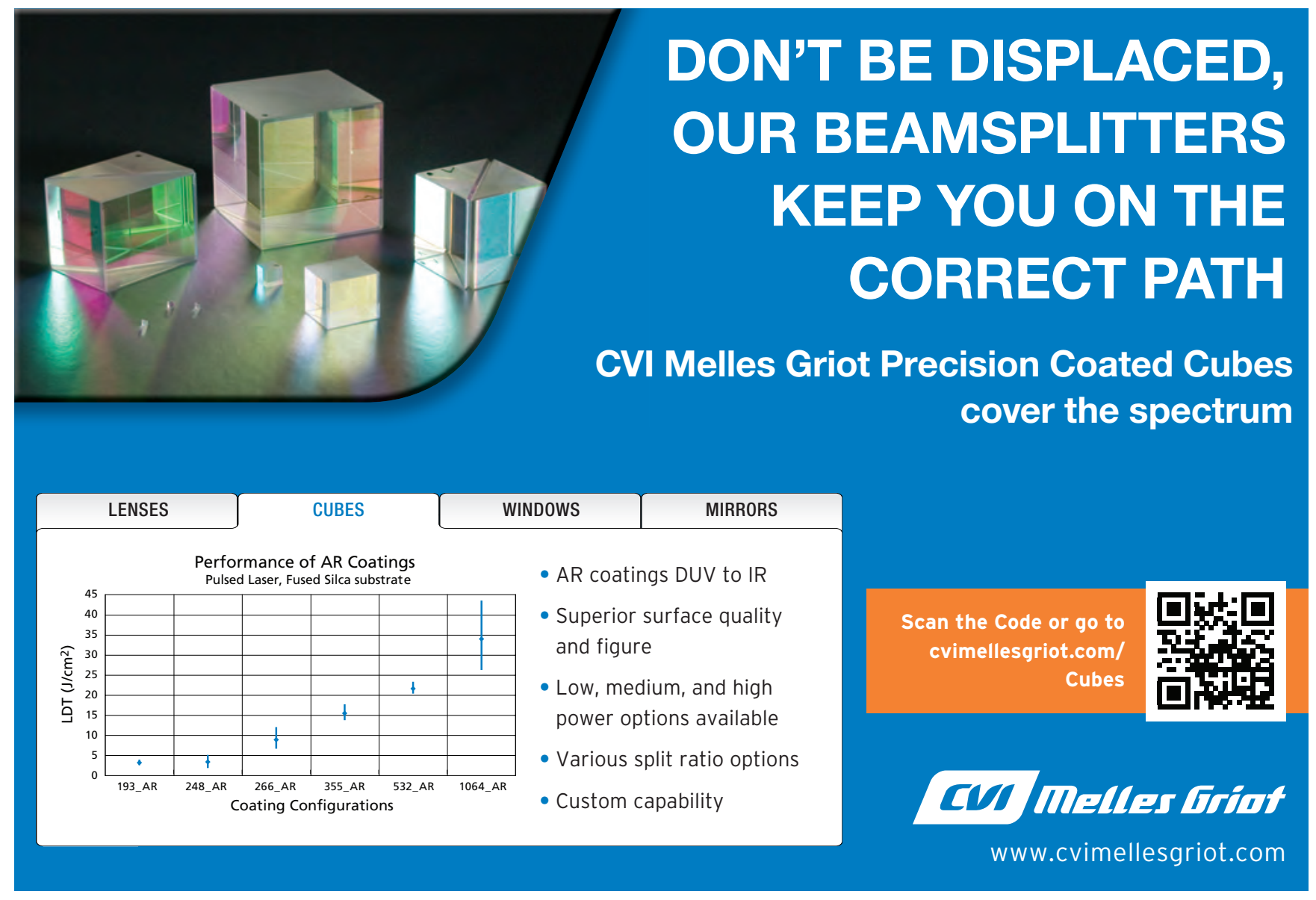

